\title{
Collisions of Planetesimals and Formation of Planets
}

\author{
Rudolf Dvorak $^{1}$, Thomas I. Maindl ${ }^{1}$, Áron Süli ${ }^{2}$, Christoph M. \\ Schäfer ${ }^{3}$, Roland Speith ${ }^{4}$, and Christoph Burger ${ }^{1}$ \\ ${ }^{1}$ Department of Astrophysics, University of Vienna, Austria \\ email: rudolf.dvorak@univie.ac.at \\ ${ }^{2}$ Eötvös University, Department of Astronomy, Budapest, Hungary \\ ${ }^{3}$ Institut für Astronomie und Astrophysik, Eberhard Karls Universität Tübingen, Germany \\ ${ }^{4}$ Physikalisches Institut, Eberhard Karls Universität Tübingen, Germany
}

\begin{abstract}
We present preliminary results of models of terrestrial planet formation using on the one hand classical numerical integration of hundreds of small bodies on CPUs and on the other hand - for comparison - the results of our GPU code with thousands of small bodies which then merge to larger ones. To be able to determine the outcome of collision events we use our smooth particle hydrodynamics (SPH) code which tracks how water is lost during such events.
\end{abstract}

Keywords. methods: n-body simulations; methods: numerical; minor planets, asteroids; planets and satellites: formation; solar system: formation

\section{Introduction and scenarios}

The outcome of numerical simulations of planet formation is extremely sensitive to initial conditions like disk surface density, total mass, the initial distribution of planetesimals and planetary embryos after the gas phase in the protoplanetary disk, and the dynamical model. Hence, the resulting planetary system models and even statistical statements are highly biased by the choice of these parameters. While many results on formation of planets and their water content have been achieved, several open questions remain (e.g., Raymond et al. 2014).

Here, we present preliminary results for a dynamical model including two gas giants, Jupiter and Saturn. By SPH simulation of collision events we can deduce the outcome of collisions in terms of merging/fragmentation and water loss depending on mass and size of the bodies, collision velocity, and impact angle (Fig. 2b and Maindl et al. 2013, 2014), thereby addressing the yet unclear effect of including water transport probabilities in planet formation simulations (cf. Izidoro et al. 2014).

We take the outcome of the last phases of the Grand Tack scenario (Walsh et al. 2011) as initial conditions for our simulations. Jupiter and Saturn are in their actual positions in the Solar System and we distribute small bodies in the mass range $3 \times 10^{-9} M_{\odot}<$ $m<3 \times 10^{-7} M_{\odot}$ and semi-major axes $0.4 \mathrm{AU}<a<2.7 \mathrm{AU}$ with small inclinations and small eccentricities. Bodies initially outside $2 \mathrm{AU}$ are assumed to have a water content of 10 percent.

\section{Preliminary simulation results}

Figure 1 shows a typical result out of 30 different runs after an integration time of 5 Myr using the Lie integration method with adaptive step size (Eggl \& Dvorak 2010). We are able to include thousands of gravitating bodies instead of hundreds by using our new massively parallelized GPU code (a descendant of the one described in Süli 2013) which is up to two orders of magnitude faster than our CPU n-body integrator (cf. 

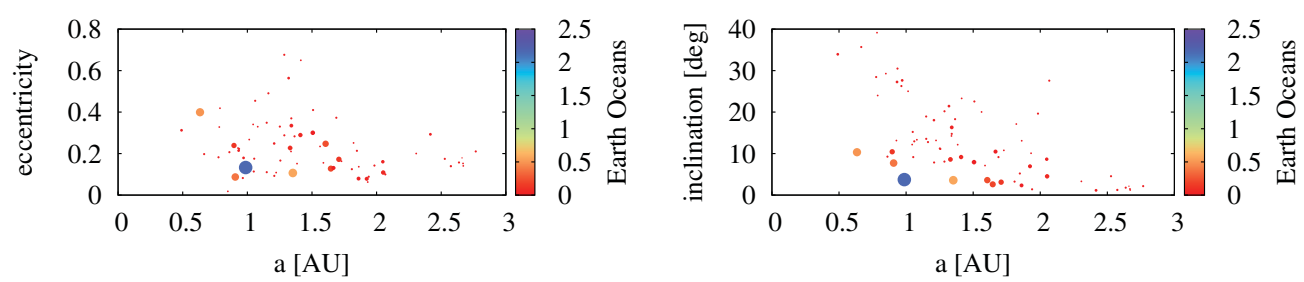

Figure 1. Eccentricities and inclinations for a typical scenario. The water content is color-coded, circle sizes denote the bodies' masses after 5 Myr. The largest bodies are $\sim 30$ lunar masses.

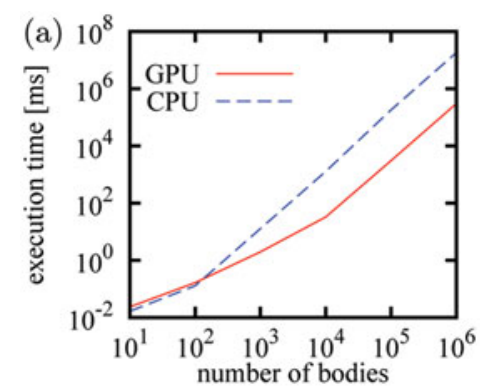

(b)

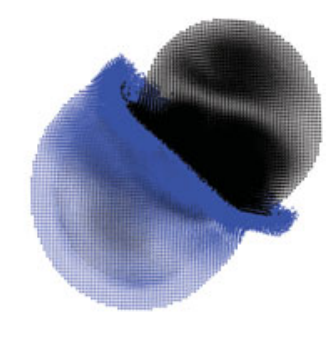

Figure 2. a) The GPU-over-CPU computational gain first grows with the number of bodies involved and settles at a factor of approximately 100. b) Low-resolution SPH simulation of two Ceres-mass bodies colliding at $1 \mathrm{~km} / \mathrm{s}$ and a $30^{\circ}$ impact angle (50k SPH particles). Blue dots: $30 \mathrm{wt}-\%$ water ice mantle on the target; black dots: solid basalt projectile.

Fig. 2a). This large number of bodies can handle dynamical friction with a precision not previously achieved (e.g., O'Brien et al. 2006).

\section{Conclusions}

While there exist several results from different groups with different underlying assumptions (e.g., Izidoro et al. 2014; Hansen 2009), we believe that independent computations including phenomena like dynamical friction and realistic collisional water transfer are desirable for answering the key questions of terrestrial planet formation.

\section{Acknowledgements}

We acknowledge support from the FWF Austrian Science Fund project S 11603-N16.

\section{References}

Eggl, S., Dvorak, R. 2010, in Lecture Notes in Physics, Berlin Springer Verlag, Vol. 790, Lecture

Notes in Physics, Berlin Springer Verlag, ed. J. Souchay \& R. Dvorak, 431-480

Hansen, B. M. S. 2009, ApJ, 703, 1131

Izidoro, A., Haghighipour, N., Winter, O. C., \& Tsuchida, M. 2014, ApJ, 782, 31

Maindl, T. I., Dvorak, R., Schäfer, C., \& Speith, R. 2014, in IAU Symposium, Vol. 310, IAU Symposium, 138-141

Maindl, T. I., Schäfer, C., Speith, R., et al. 2013, Astronomische Nachrichten, 334, 996

O’Brien, D. P., Morbidelli, A., \& Levison, H. F. 2006, Icarus, 184, 39

Raymond, S. N., Kokubo, E., Morbidelli, A., Morishima, R., \& Walsh, K. J. 2014, Protostars and Planets VI, 595

Süli, Á. 2013, Astronomische Nachrichten, 334, 1000

Walsh, K. J., Morbidelli, A., Raymond, S. N., O'Brien, D. P., \& Mandell, A. M. 2011, Nature, 475,206 